Park, Maryland 20742, USA, and in the

Department of Paleobiology, National

Museum of Natural History, Smithsonian

Institution, Washington DC, USA.

e-mail:tholtz@umd.edu

1. Lee, Y.-N. et al. Nature 515, 257-260 (2014).

2. Osmólska, H. \& Roniewicz, E. Palaeontol. Pol. 21, 5-19 (1969).

3. Rozhdestvensky, A. K. Paleontol. J. 4, 117-125 (1970).
4. Ostrom, J. H. McGraw-Hill Yearbook of Science and Technology for 1971 176-179 (McGraw-Hill, 1972)

5. Makovicky, P. J., Kobayashi, Y. \& Currie, P. J. in The Dinosauria 2nd edn (eds Weishampel, D. B., Dodson, P. \& Osmólska, H.) 137-150 (Univ. California Press, 2003).

6. Senter, P. J. Syst. Palaeontol. 5, 429-463 (2007).

7. Bell, P. R., Currie, P. J. \& Lee, Y.-N. Cret. Res. 37, 186-190 (2012).

8. Jerzykiewicz, T. in The Age of Dinosaurs in Russia and Mongolia (eds. Benton, M. J., Shishkin, M. A.,

\title{
Electron mirages in an iron salt
}

\section{The detection of unusual 'mirage' energy bands in photoemission spectra of single-atom layers of iron selenide reveals the probable cause of high-temperature superconductivity in these artificial structures. SEE LETTER P.245}

\section{JAN ZAANEN}

$\mathrm{E}$ ngineering artificial structures on the nanometre scale with the aim of controlling the quantum properties of their electrons is a mainstay of modern materials science. But it is a grand challenge to try to apply this technology to manipulate the rich, collective quantum phenomena of strongly interacting electron systems, because these systems invariably involve complicated chemistry that is hard to tame on the nanoscale. An iconic example of such phenomena is superconductivity (electrical conduction without resistance) in copper- and iron-based metallic salts at unusually high temperatures. Discovered $^{1}$ in the 1980s, this type of superconductivity is still poorly understood $d^{2,3}$, and only in the past couple of years has it become possible to manufacture high-quality single-atom layers of an iron selenide superconductor ${ }^{4}$. The surprise is that these nanolayers superconduct at higher temperatures ${ }^{4,5}$ - perhaps $^{6}$ up to a whopping 109 kelvin - than the rather poorly superconducting bulk form of this metallic salt. On page 245 of this issue, Lee et al. ${ }^{7}$ report data that seem to uncover the culprit behind this intriguing observation.

The researchers used photoemission spectroscopy to directly probe the electron worlds of iron selenide (FeSe) films, finding in their spectra highly unusual 'mirage' energy bands. It turns out that these bands can be explained in terms of physics that is unique to these nanostructures: the special nature of the interaction between the FeSe electrons and phonons (quantized crystal-lattice vibrations) in the electrically insulating strontium titanate $\left(\mathrm{SrTiO}_{3}\right)$ substrate on which the films are grown. The interaction seems to provide an ideal way of boosting the superconductivity

of the films. This result is ironic, given the contentious role of electron-phonon interactions in the long history of high-temperature superconductivity ${ }^{8}$.

Conventional superconductivity, which occurs at temperatures close to absolute zero in simple metals such as aluminium, was explained by a theory developed by John Bardeen, Leon Cooper and John Schrieffer in the mid-twentieth century. This theory describes the superconducting phase as a collective quantum state - a Bose-Einstein condensate - of 'Cooper pairs' of electrons. Cooper pairs form as a result of a quantummechanical exchange of phonons that produces

\section{The results} suggest new routes by which hightemperature superconductors can be engineered on the nanoscale. an attractive interaction between the electrons; on their own, electrons just repel each other.

However, when the news of the discovery ${ }^{1}$ of hightemperature superconductivity broke in 1986, it was clear that phonons could not be responsible for the phenomenon $^{8}$; these are already ineffective at rather low temperatures, and the repulsive interactions between the electrons are exceedingly strong in the metallic salts in which the phenomenon was observed. After nearly 30 years of struggle with intricate quantum many-body theory, eventually a consensus was reached that high-temperature superconductivity can nevertheless arise in these materials. This came about because quantum processes had been identified that could transform the strong electrostatic repulsion between the electrons into effective interactions stabilizing Cooper pairs at high temperature ${ }^{2}$.
Unwin, D. M. \& Kurochkin, E. N.) 279-296 (Cambridge Univ. Press, 2000)

9. Zanno, L. E. \& Makovicky, P. J. Proc. Natl Acad. Sci. USA 108, 232-237 (2010).

10. Zanno, L. E. \& Makovicky, P. J. Proc. R. Soc. B 280 20122526 (2013).

11.Stromer, E. Abh. König. Bayer. Akad. Wissen.

Math-Phys. Kl. 28, 1-32 (1915)

12.Ibrahim, N. et al. Science 345, 1613-1616 (2014).

This article was published online on 22 October 2014.
This electronic pairing mechanism is very different from that involving phonons ${ }^{2,8}$, to the extent that the two mechanisms have generically opposing effects in bulk metals. But according to Lee and colleagues' experiments on FeSe films, phonons in the underlying substrate can work closely together with this electronic pairing to promote superconductivity in the films.

Photoemission spectroscopy measures the probability that an electron of given energy and momentum will be removed from a sample subjected to photon excitation. In simple molecules such as hydrogen $\left(\mathrm{H}_{2}\right)$, the nature of the electron-phonon interactions is easily discerned from such spectroscopic information $^{9}$. After an electron has been removed, the molecule becomes an ion $\left(\mathrm{H}_{2}^{+}\right)$and so acquires an altered bond length. This is indicated in the photoemission spectrum as a progression of peaks: the lowest-energy peak corresponds to the pure electronic excitation, and is followed by peaks at higher energies that are associated with the emission of one, two, and so on, phonons 'repairing' the mismatch in bond length.

Lee et al. observed this spectral pattern for the specific case of the electrons in FeSe monolayers on $\mathrm{SrTiO}_{3}$ substrates. Instead of the lowest-energy peak, the authors detected an energy band that reflects the way in which the electrons quantum-mechanically delocalize in the FeSe, forming the material's metallic and superconducting states. But the authors also observed intense mirages - replicas of this 'normal' band showing up at higher excitation energies. Remarkably, this extra energy is coincident with the energy required to excite dominant phonons of the $\mathrm{SrTiO}_{3}$ substrate.

Such mirage bands have not been observed in any other solid. The reason is that in bulk metals the electron-phonon interaction becomes short-range owing to 'metallic screening' effects. As a result, the electrons excite phonons of all wavelengths, and these phonon 'shake-offs' result in featureless backgrounds in the photoemission spectrum. But in the present case, the nanometre-scale structure of the films results in the electrons exciting only phonons of long wavelength in the insulating substrate, because of unscreened interactions in this medium. This wavelength selectivity causes the appearance of mirage bands, and the strength of the mirages indicates that the electron-phonon interaction is strong. What is more, such long-wavelength 
phonons actually cooperate with the electronic-pairing mechanism ${ }^{7}$, explaining why the films superconduct at higher temperatures than their bulk analogues.

These are exciting results for superconductivity researchers, because they suggest new routes by which high-temperature superconductors can be engineered on the nanoscale. But perhaps more importantly, they are an example of the kind of surprise that might emerge from studies that attempt to harness the power of nanotechnology to explore the rich but largely uncharted territory of strongly interacting quantum systems.

Jan Zaanen is at the Instituut-Lorentz for

Theoretical Physics, Leiden University,

2300 RA Leiden, the Netherlands.

e-mail:jan@lorentz.leidenuniv.nl

1. Bednorz, J. G. \& Müller, K. A. Z. Phys. B 64, 189-193 (1986).

2. Keimer, B., Kivelson, S. A., Norman, M. R., Uchida, S. \& Zaanen, J. Preprint at http://arxiv.org/ abs/1409.4673 (2014).
3. Steward, G. R. Rev. Mod. Phys. 83, 1589-1652 (2011).

4. Wang, Q.-Y. et al. Chin. Phys. Lett. 29, 037402 (2012).

5. Liu, D. et al. Nature Commun. 3, 931; http://dx.doi. org/10.1038/ncomms1946 (2012)

6. Ge, J.-F. et al. Preprint at http://arxiv.org/ abs/1406.3435 (2014)

7. Lee, J. J. et al. Nature 515, 245-248 (2014).

8. Zaanen, J. in 100 Years of Superconductivity (eds Rogalla, H. \& Kes, P. H.) Ch. 2.4, 92-117 (Chapman \& Hall, 2011); http://arxiv.org/abs/ arXiv:1012.5461.

9. Turner, D. W. Molecular Photoelectron Spectroscopy (Wiley, 1970).

\section{Building a bigger brain}

An innovative approach to analysing the functions and gene-expression profiles of neural stem cells in developing human and mouse brains sheds light on the differences - and similarities - between the two species. SEE LETTER P. 264

\section{FORREST O. GULDEN \& NENAD ŠESTAN}

$\mathrm{T}$ The mammalian cerebral neocortex is a thin mantle of neurons on the surface of the brain's hemispheres that is largely responsible for higher-order mental functions. In different mammalian species, the cortex can vary by more than 10,000 -fold in mass ${ }^{1}$, and such variations have been associated with species-specific differences in cognition and behaviour ${ }^{1-4}$. Alterations in the proliferation and differentiation of radial glia - nonneuronal progenitor cells $\mathrm{s}^{5-7}$ that give rise to most of the approximately 16 billion neurons in the human cerebral cortex ${ }^{1}$ - are thought to play a crucial part in determining the size of the neocortex. Unfortunately, identifying the genes that regulate development of the radial glia in humans has proved exceedingly difficult. On page 264 of this issue, Lui et al. ${ }^{8}$ use a sophisticated approach to identify and characterize the role of a secreted protein, PDGFD, in mediating the proliferation of radial glia in humans.

Radial glia serve both as progenitors and as migratory guides for the daughter cells they give rise to, and so provide for the production and placement of appropriate cell numbers and types in the developing neocortex ${ }^{5-7,9}$. Changes to division of radial glia, the length of their cell cycle or the rate at which they proliferate may therefore have profound effects on the size, composition and functional repertoire of the neocortex ${ }^{2-7}$. These cells can be efficiently studied experimentally in mice and many other model organisms, but ethical and practical barriers hinder similar tests in humans. As such, genetic studies have identified many genes that both control proliferation in mice and regulate the size of the human neocortex $^{2,10,11}$, but the identities of genes involved in the development of radial glia only in humans or in non-standard laboratory animals have remained elusive.

A complete list of the genes expressed within a sample or cell type, together with information about the abundance of transcripts produced from each of these genes, is called a transcriptome. When a transcriptome is generated from a complex biological tissue such as the neocortex, it is an amalgamation of the transcriptomes of the millions or billions of cells that make up that tissue. However, cells of the same type and developmental stage tend to have more-similar transcriptomes than those of different cell types and, furthermore, cells of a given type are generally not distributed evenly throughout a tissue. Thus, it stands to reason that the expression levels of all of the genes expressed by a given cell type will rise and fall in unison as the abundance of that cell type varies in different regions of the tissue under analysis. This provides the basis for identifying the transcriptome of a single

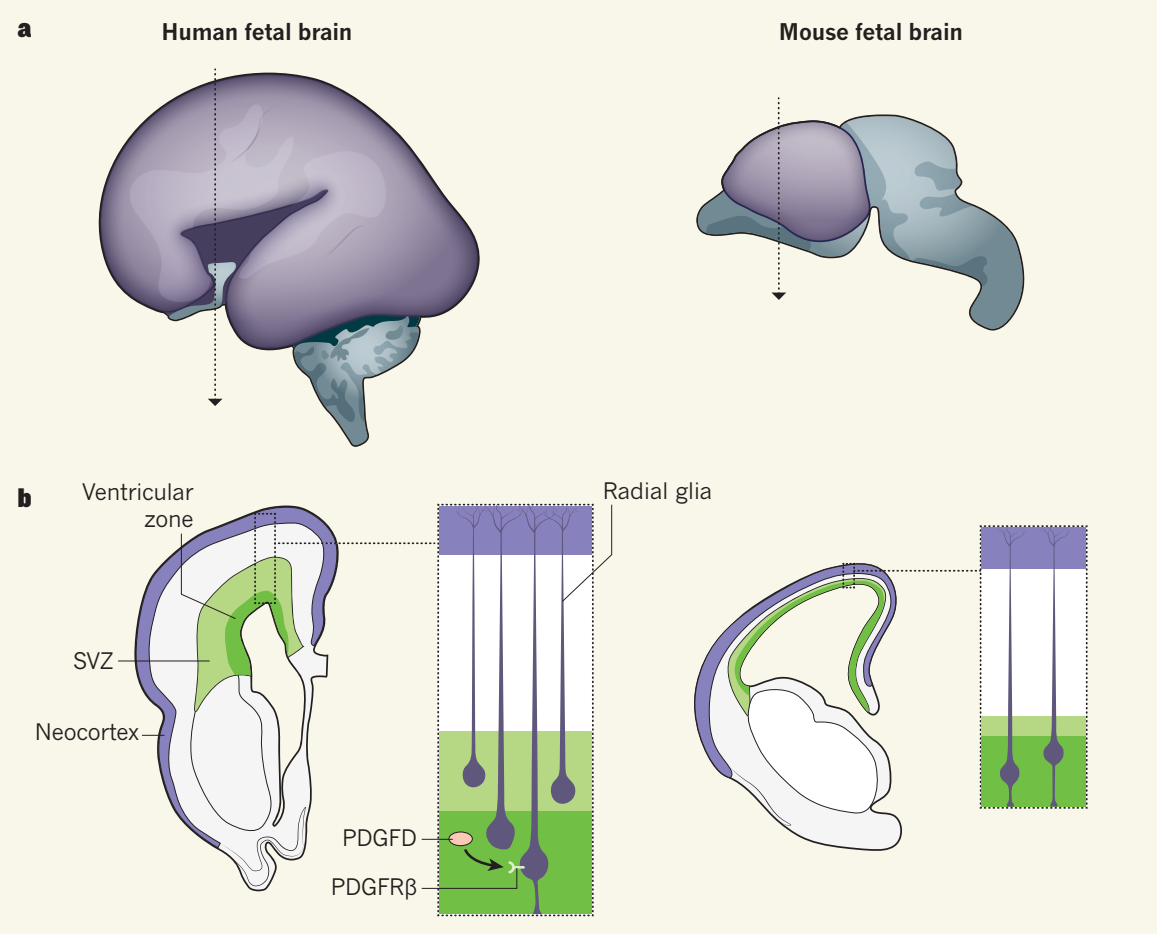

Figure 1 | Transcriptional control in radial glia. a, Side-on views of human and mouse brains at equivalent developmental stages (not to scale). The developing cerebral neocortex is indicated in purple. b, A cross-section of one brain hemisphere from each species, taken from the dotted lines shown in $\mathbf{a}$. The magnified sections show the distribution of cells in the brain called radial glia. Lui et al. ${ }^{8}$ report that the protein PDGFD, acting through the receptor protein PDGFR $\beta$, signals to the radial glia of humans but not to those of mice. PDGFD signalling correlates with increases both in proliferation of radial glia and in the distribution of cells resembling radial glia throughout the ventricular zone and subventricular zone (SVZ). 